\title{
Quadratic field extensions and residue homomorphisms of Witt rings
}

\author{
by \\ PIOTR JAWORSKI (Warszawa)
}

1. Introduction. It is well-known $[1,7]$ that with every quadratic field extension $F[z]: F, z^{2}=g$, there is associated an exact triangle of $W(F)$-modules

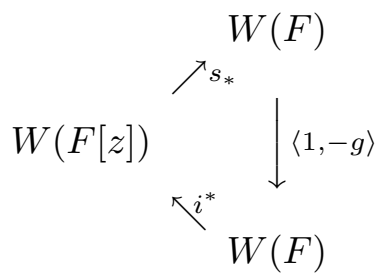

The residue homomorphisms associated with any discrete valuation $v$ on $F$ induce the morphisms of this diagram to an exact triangle of group rings

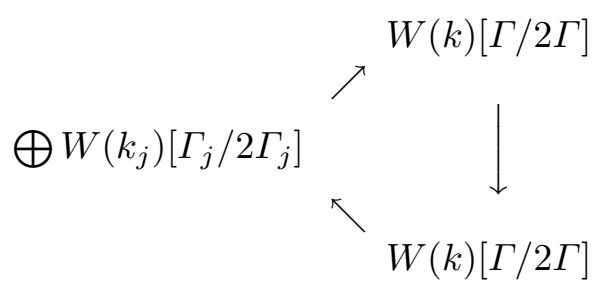

where $k$ and $k_{j}$ are the residue fields and $\Gamma$ and $\Gamma_{j}$ the value groups of $v$ and its extension (or extensions) $v_{j}$.

Using this technique we obtain several exact triangles for the Witt groups of the function fields of algebraic curves and of quadratic number fields. The first ones generalize the well-known exact sequences of Milnor and Scharlau while the second the exact sequence of the Witt groups of the field of rational numbers. Such exact triangles make it possible to reduce the problems concerning the Witt rings of function fields or quadratic number fields to

1991 Mathematics Subject Classification: Primary 11E81.

Supported by KBN, 220089102. 
the Witt rings of the residue fields. Moreover, they provide us with some new reciprocity properties.

Acknowledgements. The author would like to express his gratitude to Prof. W. Scharlau and Prof. E. Becker for helpful discussions during the preparation of the manuscript.

\section{Notation}

2.1. Homomorphisms of Witt rings. We consider the Witt rings over fields as defined in $[2,4,7]$, i.e. $W(F)$ is the Grothendieck ring of nondegenerate bilinear forms on vector spaces over the field $F$ modulo the hyperbolic forms. We recall the basic facts.

Every field homomorphism $i: K \rightarrow L$ induces a ring homomorphism of Witt rings $i^{*}: W(K) \rightarrow W(L)$. If $L / K$ is a finite field extension then every nonzero $K$-linear map $s: L \rightarrow K$ induces a transfer homomorphism $s_{*}: W(L) \rightarrow W(K)$, defined by the rule: if $\beta$ is the class containing a symmetric $L$-bilinear form $b: W \times W \rightarrow L$ then $s_{*} \beta$ is the class containing the symmetric $K$-bilinear form $s \circ b: W \times W \rightarrow K$. In this paper we shall consider the transfer map of the quadratic extension $K[\sqrt{g}] / K$ induced by the map $s, s(1)=0, s(\sqrt{g})=2$ (we assume char $K \neq 2)$ :

$$
\begin{gathered}
s_{*}: W(K[\sqrt{g}]) \rightarrow W(K), \quad s_{*}(\langle a\rangle)=0 \quad \text { for } a \in K, \\
s_{*}(\langle a \sqrt{g}+b\rangle)=\langle 2 a\rangle\left\langle 1, a^{2} g-b^{2}\right\rangle \quad \text { for } a, b \in K, a \neq 0 .
\end{gathered}
$$

We recall that $\left\langle a_{1}, \ldots, a_{n}\right\rangle$ denotes the class corresponding to the quadratic form $a_{1} T_{1}^{2}+\ldots+a_{n} T_{n}^{2}$.

The so called residue homomorphisms are other examples of mappings of Witt rings. Let $v: K \rightarrow \Gamma \cup\{\infty\}$ be a discrete valuation on the field $K$. Then $V=\{a \in K: v(a) \geq 0\}$ is a discrete valuation ring with maximal ideal $m=\{a \in K: v(a)>0\}$. Any generator $\pi$ of the ideal $m$ is called a uniformizer of the valuation. Obviously the value group $\Gamma$ is generated by the weight of $\pi$ :

$$
\Gamma=\mathbb{Z} \cdot \gamma, \quad \gamma=v(\pi) .
$$

Every element of $K$ may be uniquely written as a product $\pi^{k} a$, where $k \in \mathbb{Z}$ and $a \in V \backslash m$ (obviously $v\left(\pi^{k} a\right)=k \gamma$ ). The first and second residue homomorphisms are defined as follows:

$$
\begin{aligned}
& \partial^{i}: W(K) \rightarrow W(V / m), \quad i=1,2, \\
& \partial^{i}\left\langle\pi^{k} \cdot a\right\rangle= \begin{cases}\langle\bar{a}\rangle & \text { if } k+i \text { is odd, } \\
0 & \text { otherwise, }\end{cases}
\end{aligned}
$$

where $\bar{a}$ is the image of $a$ in the residue field $V / m$.

We remark that the residue homomorphisms are just group homomorphisms. But together they define the ring homomorphism (see [3], §7, [7], Ch. $6, \S 2)$ 


$$
\partial=\partial^{1}+\bar{\gamma} \cdot \partial^{2}: W(K) \rightarrow W(V / m)[\Gamma / 2 \Gamma]
$$

where $W(V / m)[\Gamma / 2 \Gamma]$ is the group ring and $\bar{\gamma}$ is the image of the generator $\gamma$;

$$
\partial\left(\left\langle\pi^{k} \cdot a\right\rangle\right)=\langle\bar{a}\rangle \cdot \bar{\gamma}^{\bar{k}}, \quad \bar{k} \equiv k \bmod 2 .
$$

We consider $\Gamma / 2 \Gamma$ as a multiplicative group; $\bar{\gamma}^{2}=1$.

Moreover, we remark that the above ring homomorphism and the second group residue homomorphism depend on the choice of the uniformizer $\pi$. Therefore we fix uniformizers for all valuations we consider.

2.2. Morphisms of triangles of abelian groups. We recall the following concept:

Definition. The space-diagram

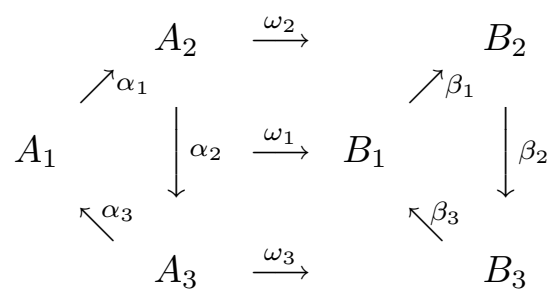

is a morphism of triangles if $\omega_{i}$ 's commute with $\alpha_{i}$ 's and $\beta_{i}$ 's:

$$
\beta_{i} \circ \omega_{i}=\omega_{j} \circ \alpha_{i}, \quad j= \begin{cases}i+1 & \text { for } i=1,2, \\ 1 & \text { for } i=3 .\end{cases}
$$

We shall use two operations on morphisms of triangles of abelian groups: the direct sum of morphisms of one triangle to other triangles:

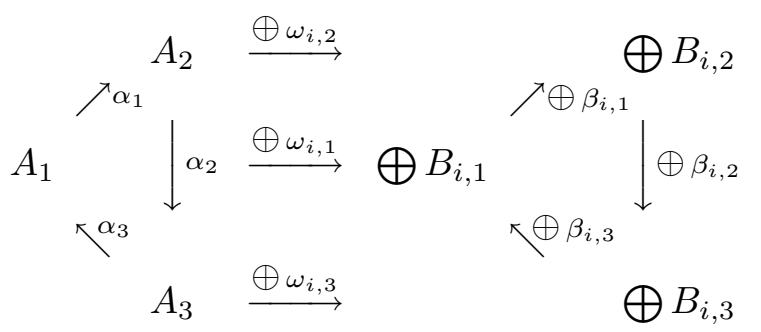

and the sum of two morphisms of two triangles to one triangle:

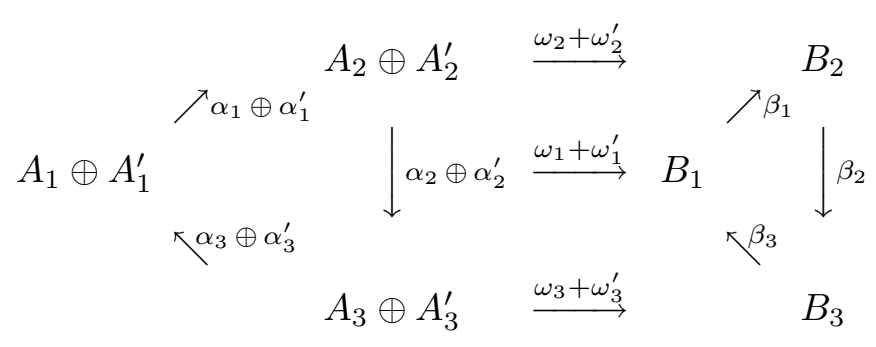


Note. We denote by the same symbol a group homomorphism defined on a summand of a direct sum and its canonical extension to a group homomorphism of the direct sum.

3. Main results. Our first theorem is a generalization of the results of $[5,6,8]$. Let $v: F \rightarrow \Gamma \cup\{\infty\}$ be a discrete valuation on the field $F$ and let $k$ be its residue field, char $k \neq 2$. We choose a uniformizer $\pi$. We shall consider the quadratic extensions $F[z]$ of $F$, where

$$
z^{2}=g, \quad g \in F \backslash F^{2}, \quad g=\pi^{n} u, \quad u \in V \backslash m .
$$

There are three cases to be considered:

Case A: $n=2 m$ and $\bar{u}$ is a square. The extension of $v$ is unramified and there are two valuations $v_{i}: F[z] \rightarrow \Gamma_{i} \cup\{\infty\}, i=1,2$, extending $v$ to $F[z]$. The value groups and the residue fields are the same as for $v$ :

$$
\Gamma_{i}=\Gamma, \quad k_{i}=k, \quad i=1,2 .
$$

Let $\delta_{i}$ be the image of $z / \pi^{m}$ in $k_{i}$. Obviously

$$
\delta_{1}=-\delta_{2} \quad \text { and } \quad \delta_{i}^{2}=\bar{u} .
$$

C a s e B: $n=2 m$ and $\bar{u}$ is not a square. The extension of $v$ is unramified; there is only one valuation $v_{1}: F[z] \rightarrow \Gamma_{1} \cup\{\infty\}$ extending $v$ to $F[z]$. The value group is the same as for $v$ but there is an extension of the residue field:

$$
\Gamma_{1}=\Gamma, \quad k_{1}=k[\bar{z}], \quad \bar{z}^{2}=\bar{u} .
$$

We denote by $\bar{i}^{*}$ and $\bar{s}_{*}$ the morphisms of Witt groups corresponding to the field extension $k[\bar{z}]: k$.

Case C: $n=2 m+1$. The extension of $v$ is ramified; there is only one valuation $v_{1}: F[z] \rightarrow \Gamma_{1} \cup\{\infty\}$ extending $v$ to $F[z]$. The residue field is the same as for $v$ but there is an extension of the value group:

$$
\Gamma_{1}=\Gamma \cdot \frac{1}{2}, \quad k_{1}=k .
$$

We let the uniformizer of the extended valuations be $\pi$ in the unramified cases $(\mathrm{A}, \mathrm{B})$ and $\widetilde{z}=z /\left(2 \pi^{m}\right)$ in the ramified case $(\mathrm{C})$.

THEOREM 1. The following diagrams are morphisms of exact triangles of $W(F)$-modules: 


$$
\begin{aligned}
& \nearrow_{s_{*}} W(F) \quad \stackrel{\partial}{\longrightarrow} \quad \nearrow_{t} W(k)[\Gamma / 2 \Gamma]
\end{aligned}
$$

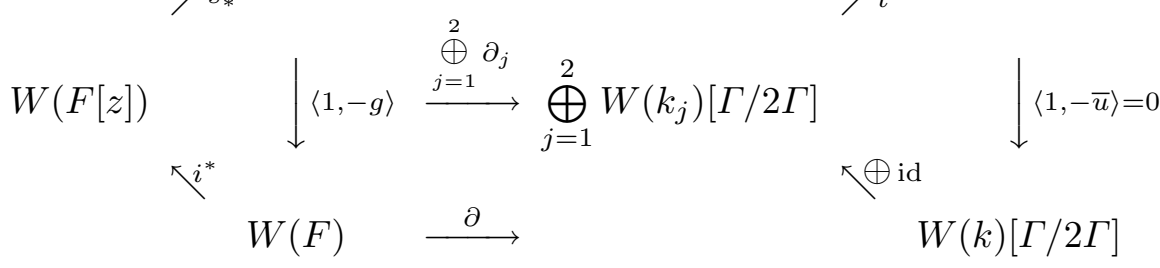

where $t\left(\alpha_{1}, \alpha_{2}\right)=\gamma^{m} \cdot\left(\sum\left\langle\delta_{i}\right\rangle \cdot \alpha_{i}\right)$, in Case A;

$$
\begin{aligned}
& W(F) \quad \stackrel{\partial}{\longrightarrow} \quad W(k)[\Gamma / 2 \Gamma]
\end{aligned}
$$

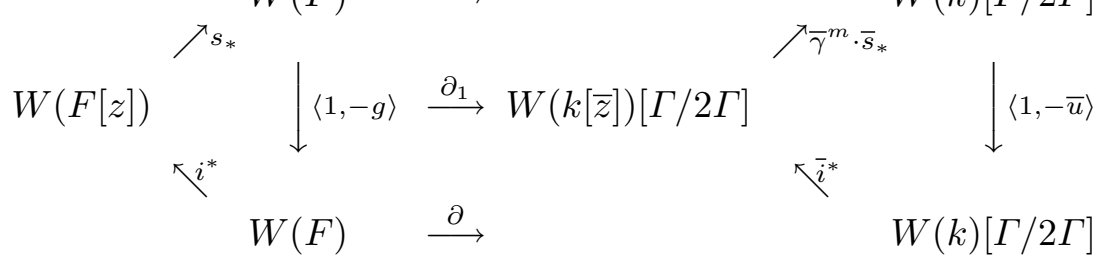

in Case B;

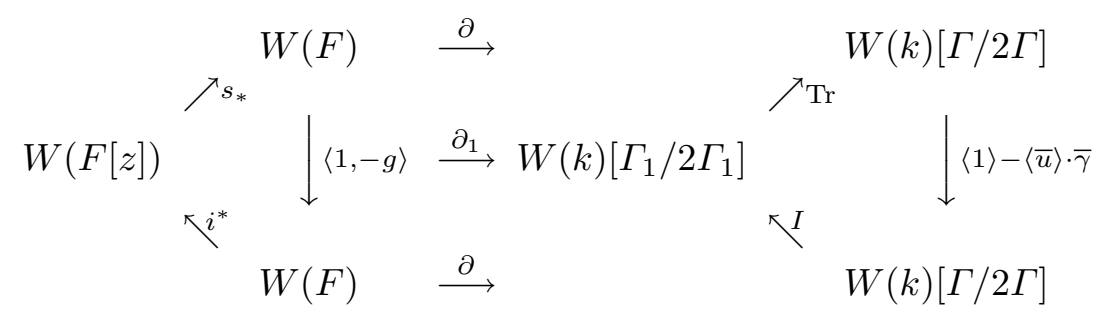

where $I(\alpha+\beta \cdot \bar{\gamma})=\alpha+\langle\bar{u}\rangle \cdot \beta$ and $\operatorname{Tr}\left(\alpha+\beta \cdot \bar{\gamma}_{1}\right)=(\langle 1\rangle+\langle\bar{u}\rangle \cdot \bar{\gamma}) \cdot \beta \cdot \bar{\gamma}^{m}$ for $\alpha, \beta \in W(k)$, in Case $\mathrm{C}$.

Remark 1. In the unramified cases (A, B), if $m$ is even (i.e. $n \equiv$ 0 mod 4) then the above morphisms of exact triangles split into the direct sum of two morphisms induced independently by the first and the second residue homomorphisms.

Some simplifications are also possible in the ramified case $(\mathrm{C})$, for example the following diagram is a morphism of exact triangles:

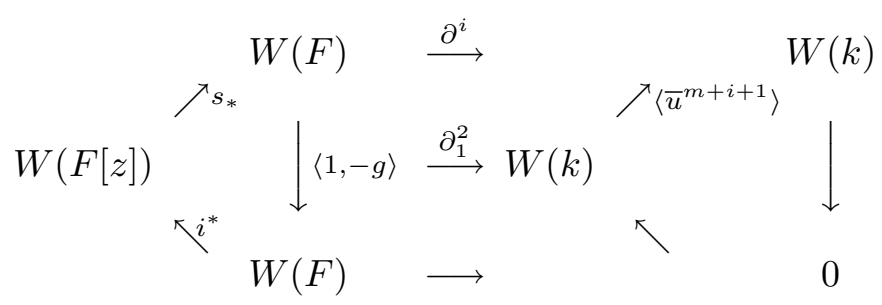

where $i=1$ or 2 . 
Remark 2. In the unramified cases (A, B), if $m$ is even (i.e. $n \equiv$ 0 mod 4$)$ then the sum $\partial^{2}+$ id induces a morphism of exact triangles:

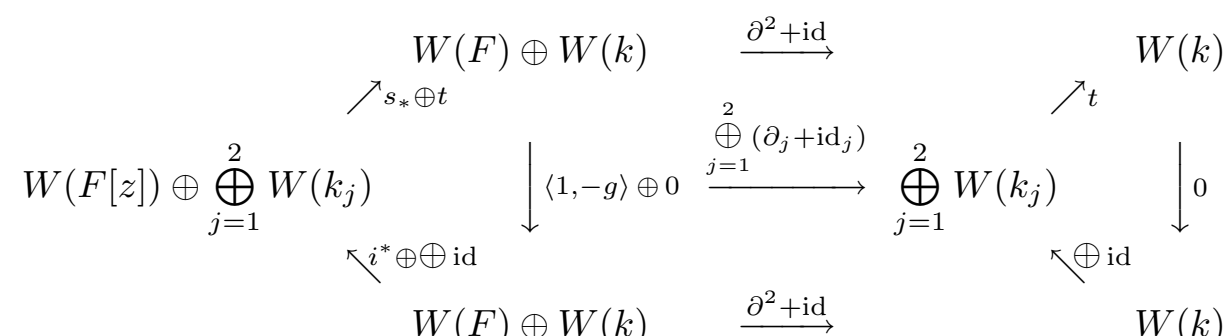

in Case A;

$$
\begin{aligned}
& W(F) \oplus W(k) \quad \stackrel{\partial^{2}+\mathrm{id}}{\longrightarrow} \quad W(k) \\
& \nearrow_{s_{*} \oplus \bar{s}_{*}} \nearrow_{\bar{s}_{*}} \\
& W(F[z]) \oplus W(k[\bar{z}]) \underset{i^{*} \oplus \bar{i}^{*}}{\downarrow} \downarrow\langle 1,-g\rangle \oplus\langle 1,-\bar{u}\rangle \stackrel{\partial_{1}^{2}+\mathrm{id}}{\longrightarrow} W(k[\bar{z}]){\overline{\bar{i}^{*}}}^{\nearrow_{\bar{s}_{*}}} \downarrow\langle 1,-\bar{u}\rangle \\
& W(F) \oplus W(k) \quad \stackrel{\partial^{2}+\mathrm{id}}{\longrightarrow} \quad W(k)
\end{aligned}
$$

in Case B.

R e m a r k 3. The residue ring homomorphism $\partial$ is the composition of the homomorphism associated with the field completion induced by the discrete valuation and the isomorphism of the Witt ring of the complete field and the group ring over the residue field. Hence the above theorem may be restated in terms of the Witt rings of the completions of the fields $F$ and $F[z]$, and the corresponding homomorphisms.

Next we consider the quadratic extension $F[z]$ of the field of rational functions in one variable: $F=K(x)$, char $K \neq 2$. We assume that

$$
z^{2}=g(x)
$$

where $g$ is a square-free nonconstant polynomial; i.e.

$$
g(x)=c \cdot g_{1}(x) \cdot \ldots \cdot g_{k}(x)
$$

where $g_{i}$ are pairwise different irreducible monic polynomials and $c$ is a nonzero constant. Let $P$ (respectively $P_{g}$ ) be the set of all valuations $v$ on the field $F$ (respectively $F[z]$ ) which are trivial on the ground field $K$ $\left(v_{\mid K}=0\right)$. The field extension $F[z]: F$ induces the mapping

$$
\chi: P_{g} \rightarrow P, \quad \chi(v)=v_{\mid F} .
$$

We split the set $P$ into the disjoint union of three subsets $A, B$ and $C$ according to the type of extension. Namely, $v \in A$ (respectively $\in B$ or $\in C$ ) 
if the extension of the valuation $v$ to $F[z]$ is unramified and split-case A above (respectively unramified and nonsplit - case B, or ramified - case C).

We fix uniformizers of the valuations in the following way:

For all $v \in P$ except the one placed at infinity, we let $\pi_{v}$ be the unique monic irreducible polynomial such that $v\left(\pi_{v}\right)>0$ (i.e. $\pi_{v}$ is the generator of the ideal $\left.v^{-1}((0, \infty)) \cap K[x]\right)$. For a uniformizer of $v_{\infty}$ we take $1 / x$.

For unramified $v \in P_{g}(\chi(v) \in A \cup B)$ we put $\pi_{v}=\pi_{\chi(v)}$. In the ramified case $(\chi(v) \in C)$ we put $\pi_{v}=z$ if $\chi(v) \neq v_{\infty}$ and, when $\operatorname{deg} g=2 m+1$, $\pi_{v}=z / x^{m}$ if $\chi(v)=v_{\infty}$.

We remark that the set $C$ is finite:

$$
\# C= \begin{cases}k & \text { if } \operatorname{deg} g \text { is even } \\ k+1 & \text { if } \operatorname{deg} g \text { is odd }\end{cases}
$$

and its elements correspond to the polynomials $g_{i}$ and, when $\operatorname{deg} g$ is odd, to "the point at the infinity".

In the following we denote by $K_{v}$ the residue field of the valuation $v$. The valuation $v$ is called simple if $K_{v}=K$.

Our next two theorems describe the exact triangles which are generalizations of the Milnor exact sequence (see [2, IX.3]).

Let $v_{1}, v_{2}$ be any simple valuations of the field $K(x)$ (i.e. $v_{i}=v_{\infty}$ or $\left.\operatorname{deg} \pi_{v}=1\right)$. We recall that the mapping

$$
\Delta_{v_{1}, v_{2}}=\partial_{v_{1}}^{1} \oplus \underset{v \in P \backslash\left\{v_{2}\right\}}{\bigoplus} \partial_{v}^{2}: W(K(x)) \rightarrow W\left(K_{v_{1}}\right) \oplus \underset{v \in P \backslash\left\{v_{2}\right\}}{\bigoplus} W\left(K_{v}\right)
$$

is a group isomorphism. We remark that the inverse of $\Delta_{v_{1}, v_{2}}$, for $v_{2}=v_{\infty}$, can be effectively computed using the filtration of the Witt group $W(K(x))$ induced by degrees of factors of coefficients (see [2, p. 266]). For other $v_{2}$ we first have to apply a projective transformation of the field $K(x)$.

We denote by $\widetilde{\Delta}_{v_{1}, v_{2}}$ the "extension" of $\Delta_{v_{1}, v_{2}}$ :

$$
\widetilde{\Delta}_{v_{1}, v_{2}}=\underset{\chi(v)=v_{1}}{\bigoplus} \partial_{v}^{1} \oplus \underset{\chi(v) \neq v_{2}}{\bigoplus} \partial_{v}^{2}: W(K(x)) \rightarrow \underset{\chi(v)=v_{1}}{\bigoplus} W\left(K_{v}\right) \oplus \underset{\chi(v) \neq v_{2}}{\bigoplus} W\left(K_{v}\right) .
$$

Moreover,

$$
\operatorname{Tr}_{v}: \bigoplus_{\chi\left(v^{\prime}\right)=v} W\left(K_{v^{\prime}}\right) \oplus W\left(K_{v^{\prime}}\right) \rightarrow W\left(K_{v}\right) \oplus W\left(K_{v}\right)
$$

is the transfer mapping (considered as a group homomorphism) associated with the valuation $v \in P$ in Theorem 1 . Let

$$
\operatorname{tr}_{v}: \bigoplus_{\chi\left(v^{\prime}\right)=v} W\left(K_{v^{\prime}}\right) \rightarrow W\left(K_{v}\right)
$$


be its (second) component:

$$
\operatorname{tr}_{v}= \begin{cases}\sum_{\bar{s}_{*}}\left(\partial_{v^{\prime}}^{1}(\langle z\rangle) \cdot\right) & \text { if } v \in A, v(g) \equiv 0 \bmod 4, \\ \partial_{v}^{2}(\langle g\rangle) \cdot & \text { if } v \in B, v(g) \equiv 0 \bmod 4, \\ \text { id } & \text { if } v \in C, v(g) \equiv 1 \bmod 4,\end{cases}
$$

TheOREM 2. The following triangle of $W(K)$-modules is exact:

$$
\begin{aligned}
& \underset{\chi(v)=v_{1}}{\bigoplus}\left(W\left(K_{v}\right) \oplus W\left(K_{v}\right)\right) \oplus \underset{\chi(v) \neq v_{1}, v_{2}}{\bigoplus} W\left(K_{v}\right) \\
& W(F[z]) \nwarrow_{i^{*} \Delta^{-1}}^{\nearrow \widetilde{\Delta}} \downarrow \downarrow \underset{v \in C \backslash\left\{v_{1}, v_{2}\right\}}{\oplus} \partial_{v}^{2}\langle 1,-g\rangle \Delta^{-1} \operatorname{tr} \\
& \bigoplus_{v \in C \backslash\left\{v_{1}, v_{2}\right\}} W\left(K_{v}\right)
\end{aligned}
$$

where $v_{1}, v_{2}$ are two different simple valuations of the field $F=K(x)$ and one of them is $v_{\infty} ; i^{*}$ is induced by $F \subset F[z]$; and

$$
\Delta=\Delta_{v_{1}, v_{2}}, \quad \widetilde{\Delta}=\widetilde{\Delta}_{v_{1}, v_{2}}, \quad \operatorname{tr}=\bigoplus_{v \neq v_{1}, v_{2}} \operatorname{tr}_{v} \oplus \operatorname{Tr}_{v_{1}} .
$$

THEOREM 3. If the degree of the polynomial $g$ is odd $(\operatorname{deg} g=2 m+1)$ then the following triangle of $W(K)$-modules is exact:

$$
\begin{gathered}
\nearrow^{\widetilde{\Delta}} \bigoplus_{v \in P_{g}} W\left(K_{v}\right) \\
\nwarrow^{i^{*} \Delta^{-1}} \\
\bigoplus_{v \in C} W\left(K_{v}\right)
\end{gathered}
$$

where $i^{*}$ is induced by $F \subset F[z]$ and

$$
\Delta=\Delta_{v_{\infty}, v_{\infty}}, \quad \widetilde{\Delta}=\bigoplus_{v \in P_{g}} \partial_{v}^{2}, \quad \operatorname{tr}=\bigoplus_{v \in P} \operatorname{tr}_{v} .
$$

We remark that the exact triangle from Theorem 3 is closely related to the exact sequence obtained in $[6]$.

Next we show how to generalize the Scharlau exact sequence (see $[2$, IX.4]). We recall that the mapping

$$
\Delta_{v_{1}, v_{2}} \oplus\left(\partial_{v_{2}}^{2}+\operatorname{id}_{W\left(K_{v_{2}}\right)}\right): W(K(x)) \oplus W\left(K_{v_{2}}\right) \rightarrow W\left(K_{v_{1}}\right) \oplus \bigoplus_{v \in P} W\left(K_{v}\right),
$$

where $v_{1}, v_{2}$ are simple valuations, is a group isomorphism. We remark that 
the inverse mapping is

$$
\begin{gathered}
\Delta_{-}: W\left(K_{v_{1}}\right) \oplus W\left(K_{v_{2}}\right) \oplus \underset{v \neq v_{2}}{\bigoplus} W\left(K_{v}\right) \rightarrow W(K(x)) \oplus W\left(K_{v_{2}}\right), \\
\Delta_{-}(\alpha, \beta, \gamma)=\Delta_{v_{1}, v_{2}}^{-1}(\alpha, \gamma) \oplus(\beta+e \cdot s(\gamma)),
\end{gathered}
$$

where $s$ is a sum of transfer mappings as defined in [2, IX.4], and $e=-1$ if $v_{2}=v_{\infty}$ and 1 otherwise.

THEOREM 4. The following triangle of $W(K)$-modules is exact:

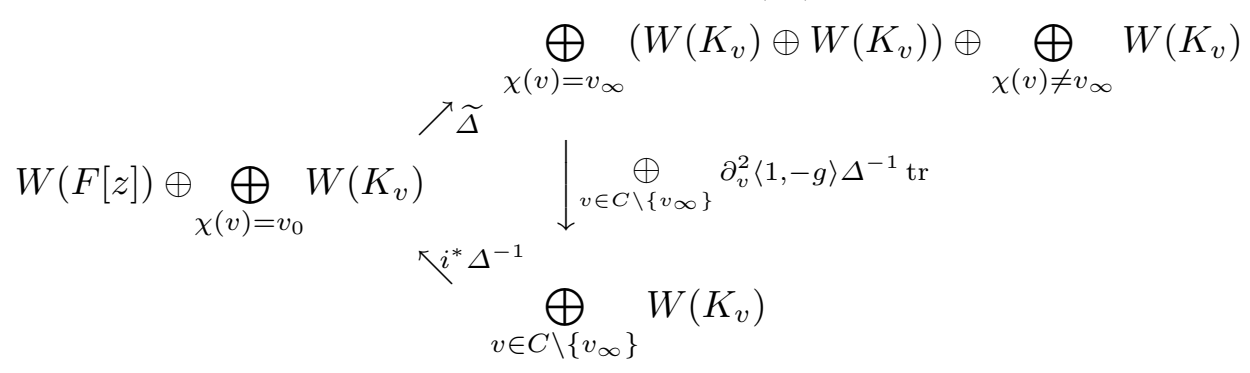

where $v_{0}$ is a simple valuation of the field $F=K(x)$ of type $A$ or $B$, and $v_{0} \neq v_{\infty}$ when $\operatorname{deg} g \not \equiv 0 \bmod 4 ; i^{*}$ is induced by $F \subset F[z] ;$ and

$$
\begin{gathered}
\Delta=\Delta_{v_{\infty}, v_{0}} \oplus\left(\partial_{v_{0}}^{2}+\operatorname{id}_{W\left(K_{v_{0}}\right)}\right), \quad \widetilde{\Delta}=\widetilde{\Delta}_{v_{\infty}, v_{0}} \oplus \underset{\chi(v)=v_{0}}{\bigoplus}\left(\partial_{v}^{2}+\operatorname{id}_{W\left(K_{v}\right)}\right), \\
\operatorname{tr}=\bigoplus_{v \neq v_{\infty}} \operatorname{tr}_{v} \oplus \operatorname{Tr}_{v_{\infty}} .
\end{gathered}
$$

Theorem 2 has a number theoretic analogue. It is well-known that the mapping

$$
\Delta=j^{*} \oplus \bigoplus_{p \in P} \partial_{p}^{2}: W(\mathbb{Q}) \rightarrow W(\mathbb{R}) \oplus \bigoplus_{p \in P} W\left(\mathbb{F}_{p}\right),
$$

where $P$ is the set of all prime numbers and $j: \mathbb{Q} \rightarrow \mathbb{R}$ is the canonical imbedding, is a homomorphism of Witt groups (see $[2,4,7]$ ).

Let $g$ be a square-free integer, $g \not \equiv 5 \bmod 8$. We consider the quadratic extension $\mathbb{Q}[\sqrt{g}]$ of the field of rational numbers. Let $j_{i}$ be the extensions of $j$.

For $g>0$ we have to consider two imbeddings

$$
j_{i}: \mathbb{Q}[\sqrt{g}] \rightarrow \mathbb{R}, \quad f_{1}(\sqrt{g})>0, \quad f_{2}(\sqrt{g})<0 .
$$

For $g<0$ we choose one,

$$
j_{3}: \mathbb{Q}[\sqrt{g}] \rightarrow \mathbb{C} .
$$

We split the set $P$ into the disjoint union of three subsets $A, B$ and $C$. We say that the prime number $p$ belongs to $A$ (respectively $B, C$ ) if the extension of the $p$-adic valuation is split unramified (respectively nonsplit unramified or ramified). We remark that $p$ belongs to $C$ if and only if $p$ 
divides $g$ or $p=2$ and $g \equiv 3 \bmod 4$. Let $P_{g}$ be the set of all extensions of $p$-adic valuations to $\mathbb{Q}[\sqrt{g}]$.

We let the prime number $p$ be a uniformizer of the $p$-adic valuation on $\mathbb{Q}$ and apply, for $p \neq 2$, the same notation as before.

For $p=2$ we define the transfer map $\operatorname{tr}_{2}$ in the following way: If $g \equiv$ 1 mod 8 then the 2 -adic valuation splits and we put

$$
\operatorname{tr}_{2}: W\left(\mathbb{Z}_{2}\right) \oplus W\left(\mathbb{Z}_{2}\right) \rightarrow W\left(\mathbb{Z}_{2}\right), \quad(\alpha, \beta) \rightarrow \alpha+\beta .
$$

If $g \equiv 0 \bmod 2$ or $g \equiv 3 \bmod 4$ then the extension is ramified and we put

$$
\operatorname{tr}_{2}=\mathrm{id}: W\left(\mathbb{Z}_{2}\right) \rightarrow W\left(\mathbb{Z}_{2}\right) \text {. }
$$

We remark that $W\left(\mathbb{Z}_{2}\right)=\mathbb{Z}_{2}$. Moreover, for $g>0$ we put

$$
\operatorname{tr}_{\infty}: W(\mathbb{R}) \oplus W(\mathbb{R}) \rightarrow W(\mathbb{R}), \quad(\alpha, \beta) \rightarrow\langle\sqrt{g}\rangle(\alpha-\beta) ;
$$

and for $g<0$,

$$
\operatorname{tr}_{\infty}=0: W(\mathbb{C}) \rightarrow W(\mathbb{R}) .
$$

TheOREM 5. The following triangles of Witt groups are exact:

$$
\begin{aligned}
& W(\mathbb{R}) \oplus W(\mathbb{R}) \oplus \bigoplus_{v \in P_{g}} W\left(K_{v}\right) \\
& \nearrow \widetilde{\Delta} \\
& W(\mathbb{Q}[\sqrt{g}]) \quad \downarrow_{p \in C}^{\oplus} \partial_{p}^{2}\langle 1,-g\rangle \Delta^{-1} \operatorname{tr} \\
& \nwarrow^{*} \Delta^{-1} \\
& \bigoplus_{p \in C} W\left(\mathbb{F}_{p}\right)
\end{aligned}
$$

for $g$ positive, and

$$
\begin{gathered}
\nearrow^{\widetilde{\Delta}} W(\mathbb{C}) \oplus \underset{v \in P_{g}}{\bigoplus} W\left(K_{v}\right) \\
\nwarrow^{i^{*} \Delta^{-1}} \\
\end{gathered}
$$

for $g$ negative. Here $i^{*}$ is induced by $\mathbb{Q} \subset \mathbb{Q}[\sqrt{g}]$, and

$$
\widetilde{\Delta}=\left\{\begin{array}{ll}
j_{1}^{*} \oplus j_{2}^{*} \oplus \bigoplus_{v \in P_{g}} \partial_{v}^{2} & \text { for } g>0, \\
j_{3}^{*} \oplus \bigoplus_{v \in P_{g}} \partial_{v}^{2} & \text { for } g<0,
\end{array} \quad \operatorname{tr}=\operatorname{tr}_{\infty} \oplus \bigoplus_{p \in P} \operatorname{tr}_{p} .\right.
$$

4. Proof of Theorem 1. The crucial point of the proof is to consider the completion $\widehat{F}$ of the field $F$ with respect to the discrete valuation $v$. 
We remark that the polynomial $z^{2}-g$ describing the field extension $F[z] / F$ may no longer be irreducible after completion. This happens in Case A (unramified, split extension), since $g$ is then a square in $\widehat{F}$. In the other cases the above polynomial remains irreducible.

More generally, let $G$ be any field extension of $F$, and $j: F \rightarrow G$.

Case 1: The equation $z^{2}-g=0$ has solutions in $G$, say $z_{1}, z_{2}$. Let $j_{i}: F[z] \rightarrow G$ be the canonical imbeddings $j_{i}(z)=z_{i}, i=1,2$.

Proposition 1. The following diagram is a morphism of exact triangles of $W(F)$-modules:

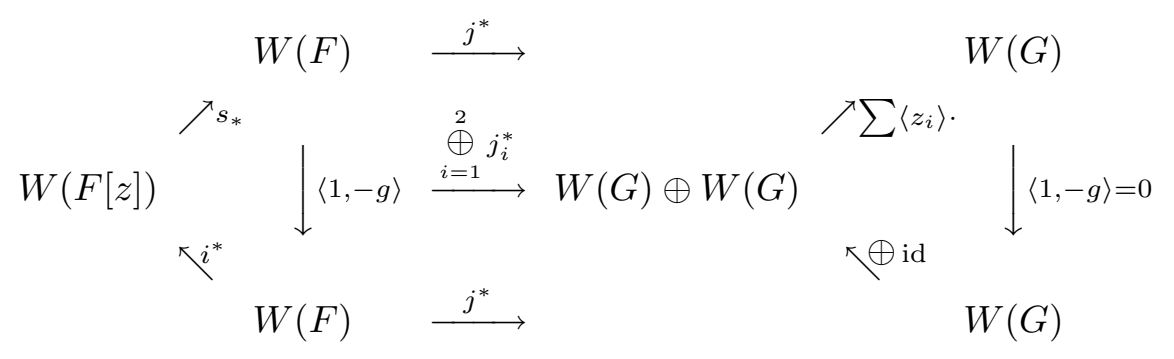

Proof. We check the commutations.

(i) $j^{*} \circ s_{*}=\sum_{i=1}^{2}\left\langle z_{i}\right\rangle \cdot j_{i}^{*}$. We check this on the generators of the $W(F)$ module $W(F[z])$ :

$$
\begin{gathered}
s_{*}(\langle 1\rangle)=0, \quad \sum_{i=1}^{2}\left\langle z_{i}\right\rangle \cdot j_{i}^{*}(\langle 1\rangle)=\sum_{i=1}^{2}\left\langle z_{i}\right\rangle=0 ; \\
j^{*} \circ s_{*}(\langle z-c\rangle)=j^{*}\left(\langle 2\rangle \cdot\left\langle 1, g-c^{2}\right\rangle\right)=\langle 2\rangle\left\langle 1, g-c^{2}\right\rangle, \\
\sum_{i=1}^{2}\left\langle z_{i}\right\rangle \cdot j_{i}^{*}(\langle z-c\rangle)=\sum_{i=1}^{2}\left\langle z_{i}\right\rangle\left\langle z_{i}-c\right\rangle=\left\langle g-c z_{1}, g-c z_{2}\right\rangle \\
=\langle 2 g\rangle \cdot\left\langle 1, g^{2}-g c^{2}\right\rangle=\langle 2\rangle\left\langle 1, g-c^{2}\right\rangle .
\end{gathered}
$$

The other commutations are obvious.

(ii) $j_{i}^{*} \circ i^{*}=j^{*}$.

(iii) $j^{*}$ is a ring homomorphism hence it commutes with multiplication.

Case 2: The equation $z^{2}-g=0$ has no solutions in $G$. We consider the field extension $G[z]: G, z^{2}=g$. Let $j_{3}: F[z] \rightarrow G[z], j_{3}(z)=z$, be the extension of $j$. The exact triangle of a quadratic extension is functorial, hence:

Proposition 2. The following diagram is a morphism of exact triangles of $W(F)$-modules: 


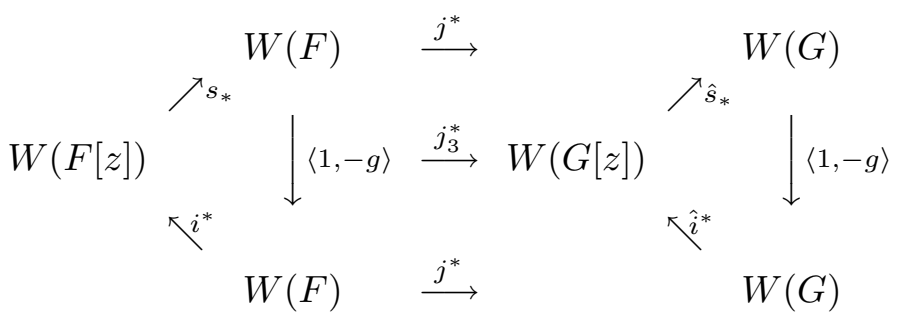

This completes the proof of Theorem 1 since the residue ring homomorphism is a ring isomorphism for complete valuations when the characteristic of the residue field is different from 2 (see $[3,7]$ ). We only have to notice that in the nonsplit cases $(\mathrm{B}, \mathrm{C})$ the transfer mapping has been changed hence we have to multiply it by a proper scaling factor (namely $\bar{\gamma}^{m}$ ). We check the formula for the trace mapping in Case C. It is enough to consider the generators $\langle 1\rangle,\langle 1\rangle \cdot \bar{\gamma}_{1}$ :

$$
\begin{gathered}
\operatorname{Tr}(\langle 1\rangle)=\partial \circ \widehat{s}_{*}(\langle 1\rangle)=0 \\
\operatorname{Tr}\left(\langle 1\rangle \cdot \bar{\gamma}_{1}\right)=\partial \circ \widehat{s}_{*}(\langle\widetilde{z}\rangle)=\partial \circ \widehat{s}_{*}\left(\left\langle\frac{z}{2 \pi^{m}}\right\rangle\right) \\
=\partial\left(\left\langle 2 \cdot \frac{1}{2 \pi^{m}}\right\rangle \cdot\left\langle 1, u \pi^{2 m+1}\right\rangle\right)=(\langle 1\rangle+\langle\bar{u}\rangle \cdot \bar{\gamma}) \cdot \bar{\gamma}^{m} .
\end{gathered}
$$

5. The Snake Lemma. The crucial point of the proofs of Theorems 2-4 is to consider the direct sum of morphisms of exact triangles and to construct a snake type homomorphism from the cokernel to kernel side.

Lemma 1 (The Snake Lemma). Let $A_{i}, B_{i}, \operatorname{ker}_{i}$ and $\operatorname{coker}_{i}$ be abelian groups, and $\alpha_{i}, \beta_{i}, \omega_{i}, \pi_{i}, j_{i}$ and $j_{-}$be group homomorphisms such that the following diagram is commutative:

$$
\begin{aligned}
& 0 \longrightarrow \operatorname{ker}_{3} \stackrel{j_{3}}{\longrightarrow} A_{3} \stackrel{\omega_{3}}{\longrightarrow} B_{3} \longrightarrow 0 \\
& 0 \longrightarrow \operatorname{ker}_{1} \stackrel{j_{1}}{\longrightarrow} A_{1} \stackrel{\omega_{1}}{\longrightarrow} B_{1} \stackrel{\pi_{1}}{\longrightarrow} \operatorname{coker}_{1} \longrightarrow 0
\end{aligned}
$$

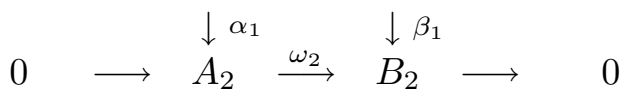

$$
\begin{aligned}
& \downarrow \alpha_{2} \quad \downarrow \beta_{2}
\end{aligned}
$$

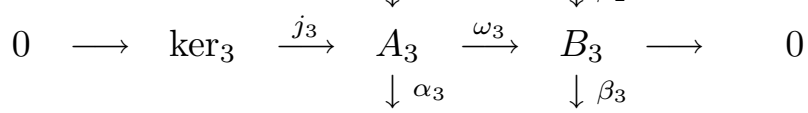

$$
\begin{aligned}
& 0 \longrightarrow \operatorname{ker}_{1} \stackrel{j_{1}}{\longrightarrow} A_{1} \stackrel{\omega_{1}}{\longrightarrow} B_{1} \stackrel{\pi_{1}}{\longrightarrow} \operatorname{coker}_{1} \longrightarrow 0 \\
& \downarrow \alpha_{1} \quad \downarrow \beta_{1} \\
& 0 \quad \longrightarrow \quad A_{2} \stackrel{\omega_{2}}{\longrightarrow} B_{2} \longrightarrow 0
\end{aligned}
$$


Moreover, suppose that the horizontal and vertical lines are exact, the $A_{3} / B_{3}$ sequence splits and $j_{-}$is the left inverse of $j_{3}$. Then:

1) the sequence

$$
0 \longrightarrow \operatorname{coker}_{1} \stackrel{\gamma}{\longrightarrow} \operatorname{ker}_{3} \stackrel{\delta}{\longrightarrow} \operatorname{ker}_{1} \rightarrow 0
$$

is exact, where

$$
\begin{aligned}
& \gamma(a)=j_{-} \circ \alpha_{2} \circ \omega_{2}^{-1} \circ \beta_{1}\left(\pi_{1}^{-1}(a)\right), \\
& \delta(a)=j_{1}^{-1}\left(\alpha_{3} \circ j_{3}(a)\right) .
\end{aligned}
$$

2) the triangle

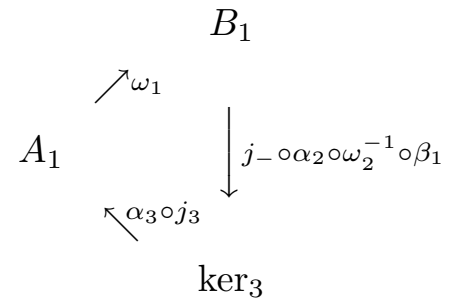

is exact.

Proof. First we show that $\gamma$ and $\delta$ are well-defined functions. Let $a=\pi_{1}(b)$. Then $\pi_{1}^{-1}(a)=b+\omega_{1}\left(A_{1}\right)$. Hence (since $\left.\alpha_{2} \circ \alpha_{1}=0\right)$ we obtain

$$
\begin{aligned}
j_{-} \circ \alpha_{2} \circ \omega_{2}^{-1} \circ \beta_{1}\left(\pi_{1}^{-1}(a)\right) & =j_{-} \circ \alpha_{2} \circ \omega_{2}^{-1} \circ \beta_{1}\left(b+\omega_{1}\left(A_{1}\right)\right) \\
& =j_{-} \circ \alpha_{2}\left(\omega_{2}^{-1} \circ \beta_{1}(b)+\alpha_{1}\left(A_{1}\right)\right) \\
& =j_{-}\left(\alpha_{2} \circ \omega_{2}^{-1} \circ \beta_{1}(b)+\alpha_{2} \circ \alpha_{1}\left(A_{1}\right)\right) \\
& =j_{-} \circ \alpha_{2} \circ \omega_{2}^{-1} \circ \beta_{1}(b) .
\end{aligned}
$$

Therefore $\gamma$ does not depend on the choice of $b$.

Since $j_{1}$ is injective we have to show that $\alpha_{3} \circ j_{3}(a)$ belongs to its image. Indeed,

$$
\omega_{1} \circ \alpha_{3} \circ j_{3}=\beta_{3} \circ \omega_{3} \circ j_{3}=0,
$$

and the kernel of $\omega_{1}$ equals the image of $j_{1}$; hence $\delta$ is well-defined.

Next we prove the exactness.

(i) $\delta \circ \gamma=0$. Indeed,

$$
\omega_{3}\left(\alpha_{2} \circ \omega_{2}^{-1} \circ \beta_{1}\right)=\beta_{2} \circ \beta_{1}=0 .
$$

Hence the image of $\alpha_{2} \circ \omega_{2}^{-1} \circ \beta_{1}$ is contained in the image of $j_{3}$ and

$$
\begin{aligned}
j_{1} \circ \delta \circ j_{-} \circ \alpha_{2} \circ \omega_{2}^{-1} \circ \beta_{1} & =\alpha_{3} \circ j_{3} \circ j_{-} \circ \alpha_{2} \circ \omega_{2}^{-1} \circ \beta_{1} \\
& =\alpha_{3} \circ \alpha_{2} \circ \omega_{2}^{-1} \circ \beta_{1}=0 .
\end{aligned}
$$

Since $j_{1}$ is injective,

$$
\delta \circ j_{-} \circ \alpha_{2} \circ \omega_{2}^{-1} \circ \beta_{1}=0 .
$$


(ii) $\operatorname{ker} \delta=\operatorname{im} \gamma$. Indeed, let $c \in \operatorname{ker} \delta$. We shall show that there exists $d \in$ coker $_{1}$ such that $\gamma(d)=c$. We have $\alpha_{3} \circ j_{3}(c)=j_{1} \circ \delta(c)=0$. Hence there exists $c_{1} \in A_{2}$ such that $\alpha_{2}\left(c_{1}\right)=j_{3}(c)$. Moreover,

$$
\beta_{2} \circ \omega_{2}\left(c_{1}\right)=\omega_{3} \circ \alpha_{2}\left(c_{1}\right)=\omega_{3} \circ j_{3}(c)=0 .
$$

Thus there exists $c_{2} \in B_{1}$ such that $\beta_{1}\left(c_{2}\right)=\omega_{2}\left(c_{1}\right)$. We put $d=\pi_{1}\left(c_{2}\right)$. Then

$$
\begin{aligned}
\gamma(d) & =\gamma \circ \pi_{1}\left(c_{2}\right)=j_{-} \circ \alpha_{2} \circ \omega_{2}^{-1} \circ \beta_{1}\left(c_{2}\right) \\
& =j_{-} \circ \alpha_{2}\left(c_{1}\right)=j_{-} \circ j_{3}(c)=c .
\end{aligned}
$$

(iii) $\delta$ is onto. Let $c \in \operatorname{ker}_{1}$. Then

$$
\alpha_{1} \circ j_{1}(c)=\omega_{2}^{-1} \circ \omega_{2} \circ \alpha_{1} \circ j_{1}(c)=\omega_{2}^{-1} \circ \beta_{1} \circ \omega_{1} \circ j_{1}=0 .
$$

Hence there exists $c_{1} \in A_{3}$ such that $\alpha_{3}\left(c_{1}\right)=j_{1}(c)$. Moreover,

$$
\beta_{3} \circ \omega_{3}\left(c_{1}\right)=\omega_{1} \circ \alpha_{3}\left(c_{1}\right)=\omega_{1} \circ j_{1}(c)=0 .
$$

Thus there exists $c_{2} \in B_{2}$ such that $\beta_{2}\left(c_{2}\right)=\omega_{3}\left(c_{1}\right)$. We put

$$
d=j_{-}\left(c_{1}-\alpha_{2} \circ \omega_{2}^{-1}\left(c_{2}\right)\right) \text {. }
$$

Then

$$
\omega_{3}\left(c_{1}-\alpha_{2} \circ \omega_{2}^{-1}\left(c_{2}\right)\right)=\omega_{3}\left(c_{1}\right)-\beta_{2}\left(c_{2}\right)=0,
$$

thus $c_{1}-\alpha_{2} \circ \omega_{2}^{-1}\left(c_{2}\right)$ belongs to the image of $j_{3}$ and

$$
\begin{aligned}
j_{1}(\delta(d)-c) & =\alpha_{3} j_{3} j_{-}\left(c_{1}-\alpha_{2} \circ \omega_{2}^{-1}\left(c_{2}\right)\right)-j_{-}(c) \\
& =\left(\alpha_{3}\left(c_{1}\right)-j_{-}(c)\right)-\alpha_{3} \circ \alpha_{2} \circ \omega_{2}^{-1}\left(c_{2}\right)=0 .
\end{aligned}
$$

Since $j_{1}$ is injective, $\delta(d)=c$.

(iv) $\gamma$ is injective. Let $c \in$ coker $_{1}$ and $\gamma(c)=0$. We shall show that for every $b \in B_{1}$ such that $\pi_{1}(b)=c$ there exists $d \in A_{1}$ such that $\omega_{1}(d)=b$. We have

$$
j_{-}\left(\alpha_{2} \circ \omega_{2}^{-1} \circ \beta_{1}(b)\right)=\gamma(c)=0
$$

and

$$
\omega_{3}\left(\alpha_{2} \circ \omega_{2}^{-1} \circ \beta_{1}(b)\right)=\beta_{2} \circ \omega_{2} \circ \omega_{2}^{-1} \circ \beta_{1}(b)=\beta_{2} \circ \beta_{1}(b)=0 .
$$

Hence $\alpha_{2} \circ \omega_{2}^{-1} \circ \beta_{1}(b)=0$ and there exists $c_{1} \in A_{1}$ such that $\alpha_{1}\left(c_{1}\right)=$ $\omega_{2}^{-1} \circ \beta_{1}(b)$. Then

$$
\beta_{1}\left(b-\omega_{1}\left(c_{1}\right)\right)=\left(\omega_{2} \circ \alpha_{1}-\beta_{1} \circ \omega_{1}\right)\left(c_{1}\right)=0 .
$$

Thus there exists $c_{2} \in B_{3}$ such that $\beta_{3}\left(c_{2}\right)=b-\omega_{1}\left(c_{1}\right)$. Since $\omega_{3}$ is onto, there exists $c_{3} \in A_{3}$ such that $\omega_{3}\left(c_{3}\right)=c_{2}$. We put $d=\alpha_{3}\left(c_{3}\right)+c_{1}$. Then

$$
\omega_{1}(d)=\omega_{1} \alpha_{3}\left(c_{3}\right)+\omega_{1}\left(c_{1}\right)=\beta_{3}\left(c_{2}\right)+\omega_{1}\left(c_{1}\right)=b .
$$

This completes the proof of the first assertion of the lemma. The second is a direct corollary of it. 
6. Exact triangles associated with algebraic curves. We remark that from the assumption that the polynomial $g(x)$ is square-free we know that for a valuation $v \in P$ there are only three possibilities:

$$
v(g)= \begin{cases}0 & \text { if } v \in A \cup B \backslash\left\{v_{\infty}\right\} \\ 1 & \text { if } v \in C \backslash\left\{v_{\infty}\right\} \\ -\operatorname{deg} g & \text { if } v=v_{\infty}\end{cases}
$$

Hence for all valuations $v \in P$ other than $v_{\infty}$, the induced morphisms of exact triangles split as in Remark 1 . To prove Theorems 2-4 one has to choose a proper set of morphisms to obtain a diagram as in the Snake Lemma.

Proof of Theorem 2. We consider the direct sum of the following morphisms: the ones induced by the second residue homomorphisms for $v \in A \cup B \backslash\left\{v_{1}, v_{2}\right\}$ :

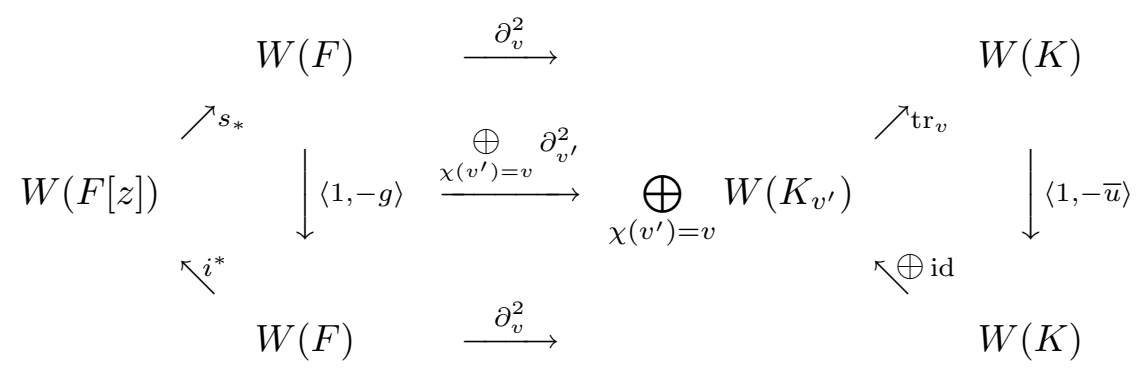

the ones shown in Remark 1 with $i=2$ for $v \in C \backslash\left\{v_{1}, v_{2}\right\}$ :

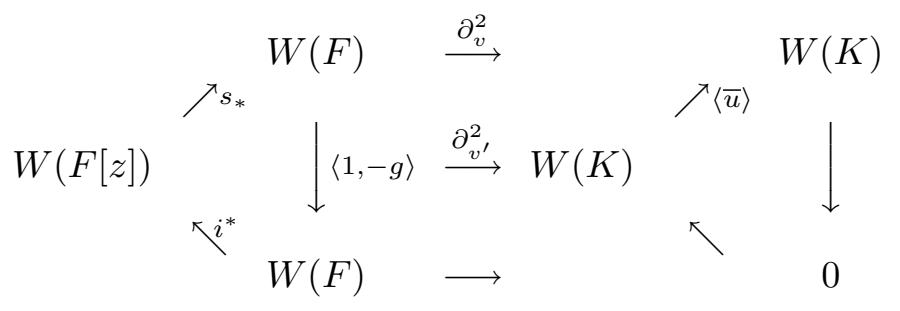

and the morphism induced by both residue homomorphisms (as in Theorem 1) for $v_{1}$.

We obtain the following commutative diagram of homomorphisms of $W(K)$-modules with vertical and horizontal lines exact: 


$$
\begin{aligned}
& \bigoplus_{\substack{v \in C \backslash\left\{v_{1}, v_{2}\right\} \\
\downarrow}} W\left(K_{v}\right) \stackrel{j}{\longrightarrow} W(F) \stackrel{\stackrel{\Delta^{\prime}}{\longrightarrow}}{\longrightarrow} W\left(K_{v_{1}}\right) \oplus W\left(K_{v_{1}}\right) \underset{\substack{v \in A \cup B \backslash\left\{v_{1}, v_{2}\right\} \\
\downarrow i^{\prime}}}{\oplus} \bigoplus_{i^{*}} W\left(K_{v}\right) \longrightarrow 0 \\
& \text { ker } \quad \longrightarrow W(F[z]) \stackrel{\tilde{\Delta}}{\longrightarrow} \underset{\chi(v)=v_{1}}{\bigoplus}\left(W\left(K_{v}\right) \oplus W\left(K_{v}\right)\right) \oplus \underset{\chi(v) \neq v_{1}, v_{2}}{\bigoplus} W\left(K_{v}\right) \\
& \downarrow s_{*} \quad \downarrow \text { tr } \\
& 0 \quad \longrightarrow W(F) \stackrel{\Delta}{\longrightarrow} W\left(K_{v_{1}}\right) \oplus W\left(K_{v_{1}}\right) \oplus \bigoplus W\left(K_{v}\right) \quad \longrightarrow 0 \\
& \downarrow\langle 1,-g\rangle \quad \downarrow m^{\prime}
\end{aligned}
$$

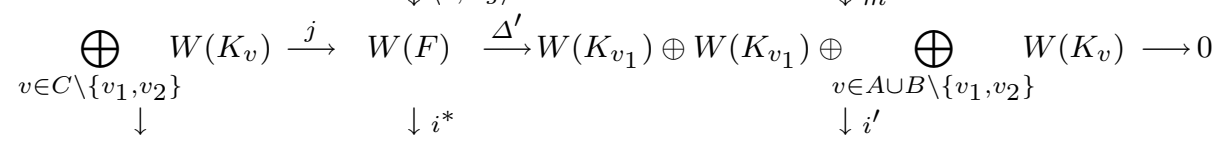

$$
\begin{aligned}
& \text { ker } \quad \longrightarrow W(F[z]) \stackrel{\tilde{\Delta}}{\longrightarrow} \underset{\chi(v)=v_{1}}{\bigoplus}\left(W\left(K_{v}\right) \oplus W\left(K_{v}\right)\right) \oplus \bigoplus_{\chi(v) \neq v_{1}, v_{2}} W\left(K_{v}\right) \\
& 0 \quad \longrightarrow \quad \stackrel{\downarrow}{s_{*}} \stackrel{\Delta}{\longrightarrow} \quad W\left(K_{v_{1}}\right) \oplus W\left(K_{v_{1}}\right) \oplus \underset{v \neq v_{1}, v_{2}}{\bigoplus} W\left(K_{v}\right) \quad \longrightarrow 0
\end{aligned}
$$

where $\Delta$ (respectively $\Delta^{\prime}, \widetilde{\Delta}$ ) is the direct sum of the second residue homomorphisms for $v \in P \backslash\left\{v_{2}\right\}$ (resp. $v \in P \backslash\left(C \cup\left\{v_{2}\right\} \backslash\left\{v_{1}\right\}\right), \chi(v) \neq v_{2}$ ) and the first residue homomorphism for $v_{1}$ (resp. $\left.v_{1}, \chi(v)=v_{1}\right)$; and $i^{\prime}, \operatorname{tr}$ and $m^{\prime}$ are compositions of the corresponding mappings of the second exact triangles (the targets).

Since $\Delta$ is a group isomorphism the kernel of $\Delta^{\prime}$ is isomorphic to the direct sum

$$
\bigoplus_{v \in C \backslash\left\{v_{1}, v_{2}\right\}} W\left(K_{v}\right),
$$

and the homomorphism $r$ is given by the restriction of the inverse mapping of $\Delta$ to the subgroup

$$
\{0\} \oplus \bigoplus_{v \in C \backslash\left\{v_{1}, v_{2}\right\}} W\left(K_{v}\right) \oplus \bigoplus_{v \in P \backslash\left(C \cup\left\{v_{2}\right\} \backslash\left\{v_{1}\right\}\right)}\{0\}
$$

of the direct sum

$$
W\left(K_{v_{1}}\right) \oplus \underset{v \neq v_{2}}{\bigoplus} W\left(K_{v}\right) .
$$

Obviously the direct sum of second residue homomorphisms

$$
\bigoplus_{v \in C \backslash\left\{v_{1}, v_{2}\right\}} \partial_{v}^{2}
$$

is a left inverse of $r$.

Pro of of Theorem 3. We consider the direct sum of the following morphisms: the ones induced by the second residue homomorphisms for $v \in A \cup B$; the ones shown in Remark 1 with $i=2$ for $v \in C \backslash\left\{v_{\infty}\right\}$; and the one shown in Remark 1 with $i=1$ for $v=v_{\infty}$. 
We obtain the following commutative diagram of homomorphisms of $W(K)$-modules with exact lines:

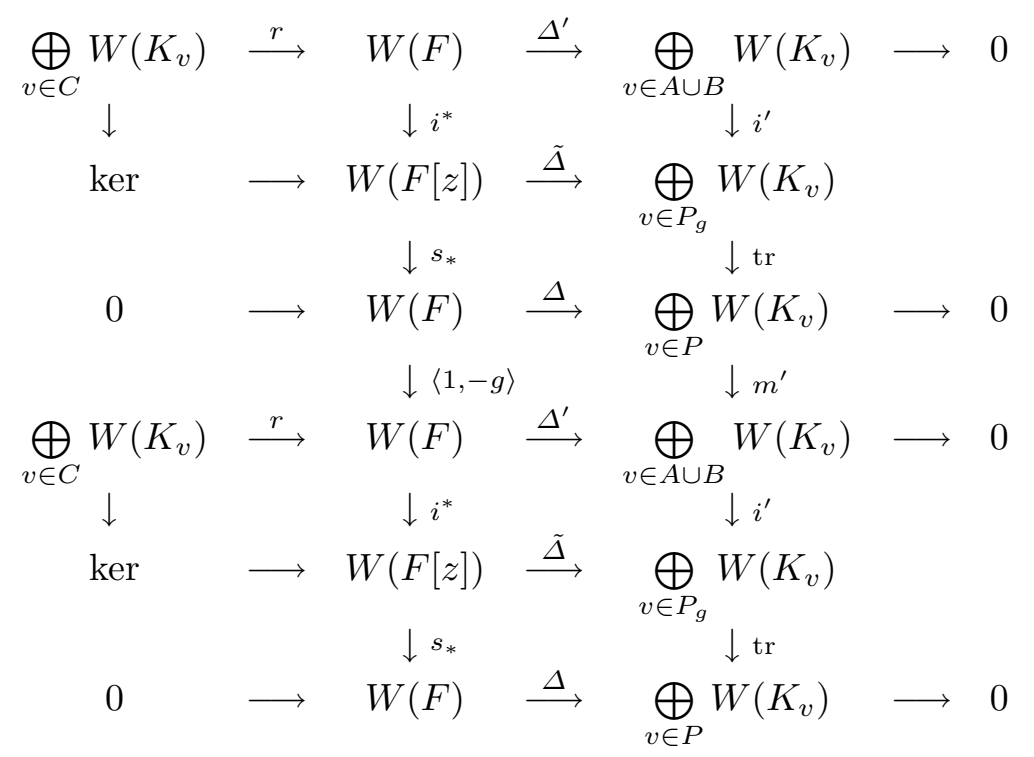

where $\widetilde{\Delta}$ (respectively $\Delta^{\prime}, \Delta$ ) is the direct sum of the second residue homomorphisms for $v \in P_{g}$ (resp. $v \in P \backslash C, v \in P \backslash\left\{v_{\infty}\right\}$ and the first residue homomorphism for $v_{\infty}$ in the last case); and $i^{\prime}$, tr and $m^{\prime}$ are compositions of the corresponding mappings of the second exact triangles (the targets).

Since $\Delta$ is a group isomorphism the kernel of $\Delta^{\prime}$ is isomorphic to $\bigoplus_{v \in C} W\left(K_{v}\right)$, and $r$ is the restriction of the inverse of $\Delta$ to the subgroup

$$
W\left(K_{\infty}\right) \oplus \underset{v \in C \backslash\left\{v_{\infty}\right\}}{\bigoplus} W\left(K_{v}\right) \oplus \underset{v \in P \backslash C}{\bigoplus}\{0\}
$$

of

$$
W\left(K_{v_{\infty}}\right) \oplus \underset{v \neq v_{\infty}}{\bigoplus} W\left(K_{v}\right)
$$

Obviously $\partial_{v_{\infty}}^{1} \oplus \bigoplus_{v \in C \backslash\left\{v_{\infty}\right\}} \partial_{v}^{2}$ is a left inverse of $r$.

Proof of Theorem 4. We consider the direct sum of the following morphisms: the ones induced by the second residue homomorphisms for $v \in A \cup B \backslash\left\{v_{0}, v_{\infty}\right\}$; the ones shown in Remark 1 with $i=2$ for $v \in C \backslash\left\{v_{\infty}\right\}$; the morphism induced by $\partial_{v_{0}}^{2}+$ id (see Remark 2); and when $v_{0} \neq v_{\infty}\left(\right.$ resp. $\left.v_{0}=v_{\infty}\right)$, the morphism induced by both residue homomorphisms (as in Theorem 1) for $v_{\infty}$ (resp. the one induced by the first residue homomorphism for $v=v_{\infty}$ ). 
We obtain the following commutative diagram of homomorphisms of $W(K)$-modules with exact lines:

$$
\begin{aligned}
& \bigoplus_{v \in C \backslash\left\{v_{\infty}\right\}} W\left(K_{v}\right) \stackrel{r}{\longrightarrow} W(F) \stackrel{\stackrel{\Delta^{\prime}}{\longrightarrow}}{\longrightarrow} W\left(K_{v_{\infty}}\right) \oplus W\left(K_{v_{\infty}}\right) \oplus \bigoplus_{\substack{v \in A \cup B \backslash\left\{v_{\infty}\right\} \\
\downarrow i^{\prime}}} W\left(K_{v}\right) \longrightarrow 0 \\
& \text { ker } \quad \longrightarrow W(F[z]) \stackrel{\tilde{\Delta}}{\longrightarrow} \bigoplus_{\chi(v)=v_{\infty}}\left(W\left(K_{v}\right) \oplus W\left(K_{v}\right)\right) \oplus \bigoplus_{\chi(v) \neq v_{\infty}} W\left(K_{v}\right) \\
& \downarrow s_{*} \quad \downarrow \text { tr } \\
& 0 \quad \longrightarrow W(F) \stackrel{\Delta}{\longrightarrow} W\left(K_{v_{\infty}}\right) \oplus W\left(K_{v_{\infty}}\right) \oplus \underset{v \neq v_{\infty}}{\bigoplus} W\left(K_{v}\right) \quad \longrightarrow 0 \\
& \downarrow\langle 1,-g\rangle \quad \downarrow m^{\prime} \\
& \bigoplus_{\substack{v \backslash\left\{v_{\infty}\right\} \\
\downarrow}} W\left(K_{v}\right) \stackrel{r}{\longrightarrow} W(F) \stackrel{\Delta^{\prime}}{\longrightarrow} W\left(K_{v_{\infty}}\right) \oplus W\left(K_{v_{\infty}}\right) \oplus \underset{\substack{v \in A \cup B \backslash\left\{v_{\infty}\right\} \\
\downarrow i^{\prime}}}{\bigoplus} W\left(K_{v}\right) \longrightarrow 0 \\
& \text { ker } \quad \longrightarrow W(F[z]) \stackrel{\tilde{\Delta}}{\longrightarrow} \bigoplus_{\chi(v)=v_{\infty}}\left(W\left(K_{v}\right) \oplus W\left(K_{v}\right)\right) \oplus \underset{\chi(v) \neq v_{\infty}}{\bigoplus_{1}} W\left(K_{v}\right) \\
& \downarrow s_{*} \quad \downarrow \text { tr } \\
& 0 \quad \longrightarrow W(F) \stackrel{\Delta}{\longrightarrow} W\left(K_{v_{\infty}}\right) \oplus W\left(K_{v_{\infty}}\right) \oplus \underset{v \neq v_{\infty}}{\bigoplus} W\left(K_{v}\right) \quad \longrightarrow 0
\end{aligned}
$$

where $\Delta$ (respectively $\Delta^{\prime}, \widetilde{\Delta}$ ) is the direct sum of the second residue homomorphisms for $v \in P \backslash\left\{v_{0}\right\}$ (resp. $v \in P \backslash\left(C \cup\left\{v_{0}\right\}\right.$ ), $\left.\chi(v) \neq v_{0}\right)$, the first residue homomorphism for $v_{\infty}$ (resp. $v_{\infty}$ and $\chi(v)=v_{\infty}$ ) and $\partial_{v_{0}}^{2}+\mathrm{id}$ (resp. $\partial_{v_{0}}^{2}+\mathrm{id}, \bigoplus_{\chi(v)=v_{0}}\left(\partial_{v}^{2}+\mathrm{id}\right)$ ); and $i^{\prime}$, tr and $m^{\prime}$ are compositions of the corresponding mappings of the second exact triangles (the targets).

Since $\Delta$ is a group isomorphism the kernel of $\Delta^{\prime}$ is isomorphic to $\bigoplus_{v \in C \backslash\left\{v_{\infty}\right\}} W\left(K_{v}\right)$, and $r$ is the restriction of the inverse of $\Delta$ to the subgroup

$$
\{0\} \oplus \underset{v \in C \backslash\left\{v_{\infty}\right\}}{\bigoplus} W\left(K_{v}\right) \oplus \underset{v \in P \backslash\left(C \backslash\left\{v_{\infty}\right\}\right)}{\bigoplus}\{0\}
$$

of

$$
W\left(K_{v_{\infty}}\right) \oplus \bigoplus_{v \in P} W\left(K_{v}\right) .
$$

Obviously $\bigoplus_{v \in C \backslash\left\{v_{\infty}\right\}} \partial_{v}^{2}$ is a left inverse of $r$.

7. Exact triangles associated with quadratic number fields. In number theoretic case one cannot avoid characteristic two.

If $g \equiv 1 \bmod 8$ then the 2 -adic valuation splits. Let $v_{1}$ and $v_{2}$ be its extensions.

Lemma 2. The following diagram is a morphism of exact triangles of Witt groups: 


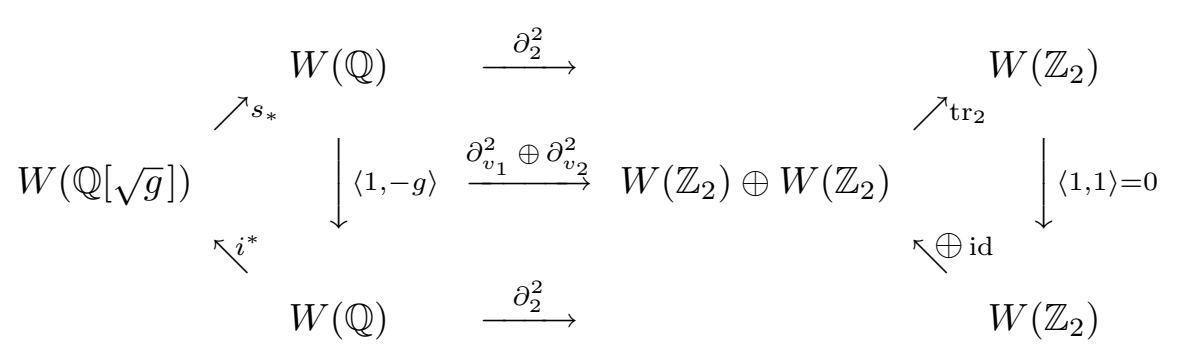

where $\operatorname{tr}_{2}(\alpha, \beta)=\alpha+\beta$.

Proof. We apply Proposition 1 for $F=\mathbb{Q}$ and $G=\mathbb{Q}_{2}$. Then we observe that the ring residue homomorphism commutes with addition and multiplication and take its second component.

If $g \equiv 3 \bmod 4$ or $g \equiv 0 \bmod 2$ then the extension $v$ of the 2 -adic valuation is ramified.

Lemma 3. The following diagram is a morphism of exact triangles of Witt groups:

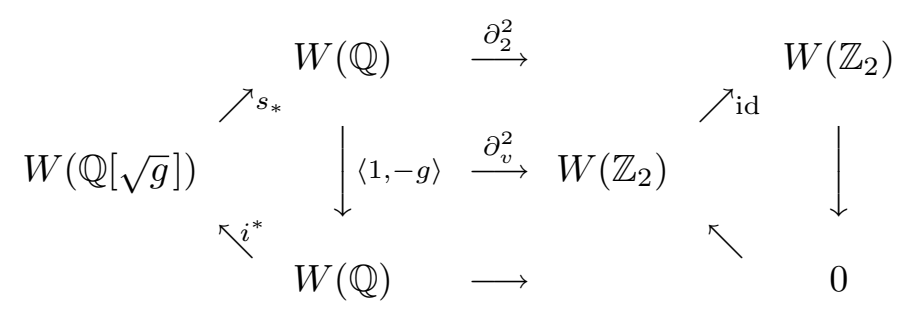

Pr o of. Let $N($ ) denote the norm of an element of $\mathbb{Q}[\sqrt{g}]$ :

$$
N(a+b \sqrt{g})=a^{2}-b^{2} g .
$$

The second residue homomorphism $\partial_{v}^{2}$ may be described in the following way:

$$
\partial_{v}^{2}(\langle c\rangle)=\partial_{2}^{2}(\langle N(c)\rangle)= \begin{cases}0 & \text { if } N(c)=4^{k}(2 l+1) \\ \langle 1\rangle & \text { otherwise }\end{cases}
$$

Hence

$$
\begin{aligned}
& \partial_{v}^{2} \circ i^{*}=0, \\
\partial_{2}^{2} \circ s_{*}(\langle a+b \sqrt{g}\rangle)= & \partial_{2}^{2}(\langle 2 b\rangle\langle 1,-N(a+b \sqrt{g})\rangle) \\
= & \begin{cases}0 & \text { if } N(a+b \sqrt{g})=4^{k}(2 l+1), \\
\langle 1\rangle & \text { if } N(a+b \sqrt{g})=2 \cdot 4^{k}(2 l+1), \\
= & \partial_{v}^{2}(\langle a+b \sqrt{g}\rangle) .\end{cases}
\end{aligned}
$$

Note. We omit the case when the extension of the 2 -adic valuation is nonsplit and unramified (this happens when $g \equiv 5 \bmod 8$ ). 
The proof of Theorem 5 is similar to that of Theorem 2. We consider the direct sum of the following morphisms: the ones induced by the second residue homomorphisms for all $p$-adic valuations; and the morphism induced by the completion of the archimedean valuation on the field of rational numbers $\mathbb{Q}, j: \mathbb{Q} \rightarrow \mathbb{R}$.

We obtain the following commutative diagram of homomorphisms of Witt groups with exact lines:

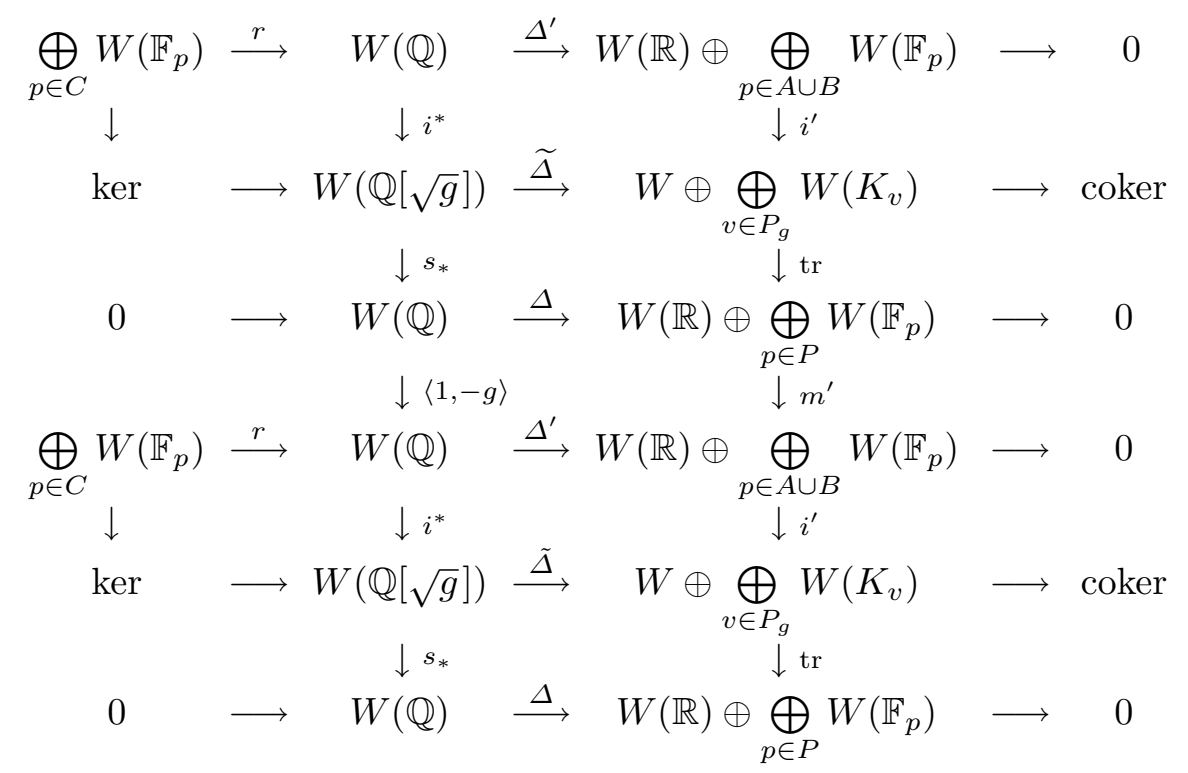

where $\Delta$ (respectively $\Delta^{\prime}, \widetilde{\Delta}$ ) is the direct sum of the second residue homomorphisms for $p \in P$ (resp. $p \in A \cup B, v \in P_{g}$ ) and the homomorphism $j^{*}$ (resp. $j^{*}, \widetilde{j}$ ); for $g>0$ we have $W=W(\mathbb{R}) \oplus W(\mathbb{R})$ and $\widetilde{j}=j_{1}^{*} \oplus j_{2}^{*}$, while for $g<0, W=W(\mathbb{C})$ and $\widetilde{j}=j_{3}^{*}$; and $i^{\prime}$, tr and $m^{\prime}$ are compositions of the corresponding mappings of the second exact triangles (the targets). Again, the kernel of $\Delta^{\prime}$ is isomorphic to $\bigoplus_{p \in C} W\left(\mathbb{F}_{p}\right)$, and $r$ is the restriction of the inverse of $\Delta$ to the subgroup

$$
\{0\} \oplus \bigoplus_{p \in C} W\left(\mathbb{F}_{p}\right) \oplus \bigoplus_{p \in A \cup B}\{0\}
$$

of $W(\mathbb{R}) \oplus \bigoplus_{p \in P} W\left(\mathbb{F}_{p}\right)$. Obviously $\bigoplus_{p \in C} \partial_{p}^{2}$ is a left inverse of $r$.

\section{References}

[1] R. Elman and T. Y. Lam, Quadratic forms under algebraic extensions, Math. Ann. 219 (1976), 21-42.

[2] T. Y. Lam, The Algebraic Theory of Quadratic Forms, Benjamin, Reading, Mass., 1973. 
[3] F. Lorenz, Quadratische Formen über Körpern, Lecture Notes in Math. 130, Springer, 1970.

[4] J. Milnor and D. Husemoller, Symmetric Bilinear Forms, Springer, Berlin 1973.

[5] R. Parimala, Witt groups of conics, elliptic and hyperelliptic curves, J. Number Theory 28 (1988), 69-93.

[6] R. Parimala and R. Sujatha, Witt groups of hyperelliptic curves, Comment. Math. Helv. 65 (1990), 559-580.

[7] W. Scharlau, Quadratic and Hermitian Forms, Springer, 1985.

[8] J. E. Shick, Quadratic forms over function-fields of elliptic and hyperelliptic curves, PhD thesis, Univ. of California, San Diego 1991.

INSTITUTE OF MATHEMATICS

UNIVERSITY OF WARSAW

BANACHA 2

02-097 WARSZAWA, POLAND 